\title{
Evaluation Model of College Students Aerobics Athlete Selection Based on Fuzzy Neural Network
}

\author{
Cong Ding \\ Department of Physical Education, North-west University, Xi’an, 710069, China \\ dddingcong@163.com
}

Keywords: College Student; Aerobics; Selection; Fuzzy Neural Network

\begin{abstract}
In order to improve the talent identification level of college students aerobic athletes. With the help of Fuzzy Transformation Theory, this paper establishes the model of selecting college aerobics athletes by neural networks. From determining the indexes of the selected team members, solving the comprehensive evaluation value to selecting qualified athletes, this paper makes a systematic study and designs the athlete based on BP neural network selecting decision support system logic model, completing a preliminary work for the physical model of the system.
\end{abstract}

\section{Introduction}

Athletes' talent is often an important basis for scientific training[1-2]. Therefore, the art of choosing athletes is the general focus of attention in sports. The competitive training of college students is an amateur wat and the college aerobics students are also amateur athletes. Compared with professional athletes, they have shorter training periods, less time, shorter exercise life and heavier competition tasks. Several problems remain to be solved, such as that the training cycle and time is short, the training program cannot increase the proportion of physical training issues. Students with better conditions in all aspects who have aerobics training base or participated in aerobics matches or physical fitness are undoubtedly the best choice. The students who have participated in aerobics training or competition can often be differentiated at the first glance. The key point is how to choose from a group of students who are full of confidence and volunteer to participate in aerobics training scientifically and with better physical quality conditions.

Athletes selection should be considered from a variety of factors, and these considerations can only be described in vague language. At this moment, fuzzy evaluation method can be used in athletes selection. Fuzzy comprehensive evaluation method is based on the determination of evaluation criteria and weights of evaluation factors, and uses the principle of fuzzy set transformation to describe the fuzzy boundaries of various factors and units by membership degree[3]. The level of evaluation object will be ultimately determined by constructing the fuzzy evaluation matrix and multilevel computation[4]. Therefore, in this study, we use Artificial neural network to build the supplementary system of the material selection for the college students aerobics athletes.

\section{Design of Athletes' Fuzzy Evaluation}

In this part, the establishment of evaluation index system is completed by organizing a certain number of experts and establishing the aerobics athletes selection physical fitness test items in college students. First, define the top-level indicator as population, then subdivide the population into several sub-items in total, so that all the participating projects will form a hierarchical index tree. The overall evaluation may be four strength indicators of flexibility strength, speed strength, burst stamina and coordination, and the flexible strength may be subdivided into longitudinal splits index, sit-ups, skipping ropes. The speed of force can be subdivided into two secondary indicators, namely 50m run and 30s abdomen lift leg. Outbreak endurance can be subdivided into three secondary indicators, namely standing long jump, 30s push-ups, $800 \mathrm{~m}$ run and coordination 
quadrant movement. In this way, an indicator hierarchy is constructed.

\section{Determine the weight vector of the index}

The influence of the indicator in the same level on indicators in the upper level is reflected as a weight vector, where a comparative matrix method is used to calculate the weight vector. We organizes the experts again to compare the indicators of each level, and compare them to get the comparison relation matrix $A=\left[a_{\mathrm{ij}}\right] \mathrm{N} \times \mathrm{N}$, where aij represents the quantitative comparison of the indicators ui and uj, the values of which refer to the numbers $1,2, \ldots, 9$ and their reciprocal 1,1 / $2, \ldots, 1 / 9$ is taken as a scale, and provides aji=1/aij, aii=1[5]. The meanings of their representatives are shown in Table 1 . Then, the eigenvector is calculated according to the root method: The Nth power root $\overline{\mathrm{pi}}$ of the product of each row in the matrix $\mathrm{A}$ is calculated, and the weight vector $\mathrm{P}$ is calculated by normalization.

Tab. 1 The meanings of $\mathrm{a}_{\mathrm{ji}}, \mathrm{u}_{\mathrm{i}}$ and $\mathrm{u}_{\mathrm{j}}$

\begin{tabular}{|c|c|}
\hline Parameter $\mathrm{a}_{\mathrm{ij}}$ & Meaning \\
\hline 1 & The ratio of the impact of $\mathrm{u}_{\mathrm{i}}$ to $\mathrm{u}_{\mathrm{j}}$ is the same \\
\hline 3 & The ratio of the impact of $\mathrm{u}_{\mathrm{i}}$ to $\mathrm{u}_{\mathrm{j}}$ is slightly superior \\
\hline 5 & The ratio of the impact of $\mathrm{u}_{\mathrm{i}}$ to $\mathrm{u}_{\mathrm{j}}$ is superior \\
\hline 7 & The ratio of the impact of $\mathrm{u}_{\mathrm{i}}$ to $\mathrm{u}_{\mathrm{j}}$ is obviously superior \\
\hline 9 & The ratio of the impact of $\mathrm{u}_{\mathrm{i}}$ to $\mathrm{u}_{\mathrm{j}}$ is ultimately superior \\
\hline $2,4,6,8$ & The ratio of the impact of $\mathrm{u}_{\mathrm{i}}$ to $\mathrm{u}_{\mathrm{j}}$ is in between adjacent levels \\
\hline $1 / 2, \ldots, 1 / 9$ & The ratio of the impact of $\mathrm{u}_{\mathrm{i}}$ to $\mathrm{u}_{\mathrm{j}}$ is opposite to each other \\
\hline
\end{tabular}

\section{Fuzzy transformation processing}

In the whole index system, it is assumed that an index has $M$ sub-indexes, which constitute the index domain $U=\{u k\} ; N$ fuzzy evaluation levels are selected to form the domain $V=\{v k\}$, and the weight vector $\mathrm{P}=[\mathrm{p} 1, \mathrm{p} 2, \ldots, \mathrm{pM}]$ of the $\mathrm{M}$ secondary indicators corresponding to the indicator can be obtained according to (1). We organized all the evaluators to make fuzzy appraisals on these M indicators and then calculate the distribution of the appraisal levels for each indicator. That is, suppose that the ratio of the number of evaluators who make level vj to the index ui to all the number of evaluators is rij, such that $\mathrm{R}=[\mathrm{rij}] \mathrm{M} \times \mathrm{N}$ constitutes the fuzzy relation on $\mathrm{U} \times \mathrm{V}$. [2] uses weight vector $\mathrm{P}$ to perform fuzzy transformation on $\mathrm{R}$, and obtains the fuzzy relation $\bar{Q}=P \circ R$ of this index on the universe V. The result of fuzzy evaluation $\mathrm{Q}$ of the index $\bar{Q}$ is normalized. You can think of it as a secondary indicator later. Repeat the previous steps in order to solve the upper level indicators of the evaluation, and ultimately get the overall fuzzy evaluation.

\section{Establishing Neural Network Structure and Training Neural Network}

Here we design a $\mathrm{N}$-input-single-output BP network to make the final score for the players who participated in the evaluation[6]. The network consists of a hidden layer with M nodes and uses the hyperbolic tangent function as the transfer function. However, the problem of how to choose the hidden layer nodes is so complicated that there exists no good analytical solution at present. The number of hidden nodes is often based on the design experience of previous research and the researchers' own experiments to determine.

We organized experts to perform ambiguous evaluations and overall scoring of individual indicators on L subjects. The fuzzy evaluation of single index is fuzzy transformed to obtain the overall fuzzy evaluation of $\mathrm{L}$ group $\mathrm{Xk}^{*}, \mathrm{k}=1,2, \ldots, \mathrm{L}$; the corresponding overall score is $\mathrm{yk}^{*}$, 
$\mathrm{k}=1,2, \ldots \mathrm{L} .\left(\mathrm{Xk} *, \mathrm{yk}^{*}\right)$ constitutes a learning sample of the BP network. According to the learning samples, the constructed neural network is trained and learned. After repeated iterations, the average error of the system is reduced to a satisfactory level so that a stable network structure can be obtained and its connection weights can be determined. Since neural network learning is a nonlinear optimization problem, it is inevitable that there exists a local optimal solution [7]. Therefore, we introduce genetic algorithm here because genetic algorithm does not directly affect the parameter set, but uses some kind of coding of the parameter variable; the search is started not from a single point but from a cluster of points; probability transfer method, rather than the rules of certainty, will be adopted; in the search process, the local optimum may be avoided. The process is shown as follows:

\subsection{Provide training samples}

According to the learning sample $\left(\mathrm{Xk}^{*}, \mathrm{yk}^{*}\right), \mathrm{k}=1,2, \ldots, \mathrm{L}$ provided by experts, the pth group of inputs is: $\mathrm{Xp}^{*}=(\mathrm{xp} 1, \mathrm{xp} 2, \ldots, \mathrm{xpm})$, the pth output is yp, where: $\mathrm{L}$ is the number of samples; in this paper, there are $\mathrm{n}$ input nodes, $\mathrm{m}$ hidden layer nodes and an output node.

\subsection{Define individual fitness function}

Suppose the input layer to the hidden layer connection has weight vector $W_{\mathrm{ij}}^{1}$, the size is $\mathrm{n} \times \mathrm{m}$, $\omega_{\mathrm{ij}}^{1}$ represents the weight of connection between input node $\mathrm{i}$ to the hidden layer node $\mathrm{j}$; suppose the connection weight vector from the hidden layer to the output layer is $W_{\mathrm{i}}{ }^{2}$, the size is $\mathrm{m} \times 1$, $\omega_{\mathrm{i}}^{2}$ represents the connection weight from the hidden node $\mathrm{i}$ to the output node. To input a sample of neural network $X_{P}^{*}=\left(x_{p 1}, x_{p 2}, \cdots, x_{p n}\right)$, the input corresponding to hidden layer node $\mathrm{i}$ $I_{\mathrm{i}}=\sum_{\mathrm{s}=1}^{\mathrm{n}} W_{\mathrm{si}}^{1} \mathrm{x}_{\mathrm{ps}}$, the output node corresponding to hidden node $\mathrm{I}$ is $O_{\mathrm{i}}=\mathrm{f}\left(I_{\mathrm{i}}\right)$. The input corresponding to output node is $I=\sum_{i=1}^{m} W_{i}^{2} o_{i}, \mathrm{i}=1 \mathrm{~W} 2 \mathrm{iOi}$, and the output of the output node is $\mathrm{O}=\mathrm{f}$ (I), so the actual output of the neural network can be obtained by calculating yp. In order to make the output of neural network close to the expected value of the sample, the goal of this problem can be transformed into the seeking the minimum type $\sum_{p=1}^{1}\left(y_{p}-y_{p}^{*}\right)^{2}$, so the fitness function should be the maximum. The fitness of the definition of individual $\mathrm{X}$ is:

$$
F(X)=\left[\sum\left(y_{p}-y_{p}^{*}\right)^{2}\right]^{-1}
$$

\subsection{Coding and the generation of initial population}

A real interval [ $\omega$ min, $\omega \max ]$ is empirically determined, and all $\omega \mathrm{ij}$ are uniformly distributed in the interval $[\omega \mathrm{min}, \omega \max ]$, chromosome $\mathrm{W}$ is generated, thus generating the initial population.

\subsection{Crossover operator}

Crossing is calculated with a certain crossover probability. We used arithmetic crossover method to cross operator because here is the real number encoding. Suppose $\omega 1$ and $\omega 2$ as the parents of

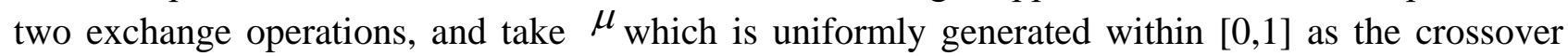
probability, then the corresponding generations $\omega^{\prime}{ }_{1}$ and $\omega^{\prime}{ }_{2}$ of the exchange operations are:

$$
\left\{\begin{array}{l}
\omega_{1}^{\prime}=\mu_{\omega_{1}}+(1-\mu)_{\omega 2} \\
\omega^{\prime}{ }_{2}=(1-\mu) \omega 1+\mu \omega 2
\end{array}\right.
$$


Equation (2) ensures that the weights among all nodes in the new weights assigned to the network are still within the interval [ $\omega$ min, $\omega$ max $]$, and $\omega_{1}+\omega_{2}=\omega_{1}^{\prime}+\omega^{\prime}{ }_{2}$ can be guaranteed.

\subsection{Mutation operator}

Assuming that the gene $\omega \mathrm{ij}$ in the parent individual $\omega$ is selected as the mutation, a random number $\omega^{\prime}{ }^{\prime}$ is generated from a uniform distribution[ $\left.\omega \min , \omega \max \right]$ to replace $\omega_{i j}$.

\subsection{Termination conditions}

Determine the minimum number of iterations in advance, and observe the change of fitness of the best individual in each generation when the number of times exceeds the specified number of times. If the fitness of the best individual is greater than a predetermined value and there is neither growth nor slow growth, the calculation will be terminated. If the condition is not satisfied, return to step (2) until the condition is satisfied.

\section{Selection of qualified candidates}

A comprehensive evaluation value can be derived from fuzzy evaluation by artificial neural network, assuming that the overall evaluation of the $x$-th student value is $f(x)$, and the comprehensive evaluation value of all the participated students can be obtained as ( $\mathrm{f}(1), \mathrm{f}(2), \ldots, \mathrm{f}$ $(\mathrm{m})$ ), where $\mathrm{m}$ is the total number of students who participated in the assessment. Students with the accordingly biggest evaluation value will be selected, and then sent to the coaching staff for final review.

According to the comprehensive evaluation value output by the neural network module, all the evaluation values are arranged in sequence, and according to the needs of college aerobics team building, a qualified student list is generated.

\section{Conclusion}

In this paper, the design of university aerobics athletes selection system has been studied. This paper initially proposed to establish model structure of systematically selecting college aerobics athletes. Also, a system module diagram is completed by analysis. After realizing the informationization, the mature of software and hardware is very important to make this system possible. An excellent decision support system needs to be supported by a good model library, and all these issues call for further research and thinking.

\section{References}

[1] Philip T, Kelly W,Diane C.Physical activity and mental health in a student population[J]. Journal of Mental Health, 2010, 11 (6):23-29.

[2] Rita K, Bode, Allen W. Course of functional improvement after stroke, spinal cord injury, and traumatic brain injury [J]. Archives of Physical Medicine and Rehabilitation, 2002, 18 (1):119-126.

[3] P F. Yan, C S. Zhang, Artificial neural network and simulated evolutionary computation [M]. Tsinghua university press, Bei Jing, 2000.

[4] Li, H., Zhang, Z., \& Liu, Z. Application of artificial neural networks for catalysis: A review [J]. Catalysts, 2017, 7(10):306-311.

[5] L M. Zhang, Artificial neural network model and its application [M]. Fudan University Press, Shang Hai, 2009.

[6] Li, H., Liu, Z., Liu, K., \& Zhang, Z.Predictive Power of Machine Learning for Optimizing Solar Water Heater Performance: The Potential Application of High-Throughput Screening[J]. International Journal of Photoenergy, 2017. 
[7] Li, H., Tang, X., Wang, R., Lin, F., Liu, Z., \& Cheng, K. Comparative Study on Theoretical and Machine Learning Methods for Acquiring Compressed Liquid Densities of 1, 1, 1, 2, 3, 3, 3-Heptafluoropropane (R227ea) via Song and Mason Equation, Support Vector Machine, and Artificial Neural Networks[J]. Applied Sciences, 2016, 6(1):17-25. 